\title{
THE TREATMENT OF MYELOMENINGOCELE PATIENTS WITH PARALYTIC HIP DEFORMITIES BY ILIOPSOAS TRANSFER
}

\author{
By Alvin A. Freehafer, M.D. \\ Division of Orthopaedic Surgery, Case Western Reserve University School of Medicine, \\ University Hospital and Cuyahoga County Hospitals, Cleveland, Ohio
}

AN increasing number of patients with myelomeningocele of the lumbo-sacral spine come under medical care each year. This is probably directly related to the improvements in treatment leading to control of hydrocephalus, early closure of the spinal defect and improved care of the genito-urinary system.

Many of these children have sufficient intelligence and potential for rehabilitation. Because of this the orthopaedist is playing an increasingly important role in the prevention and correction of deformities so that these children can attain a maximum functional level.

Problems occurring at the hip joints of these patients represent only one small area of concern for physicians caring for these patients. However, the importance of good hip function in severely paralysed persons cannot be underestimated. If left untreated, some will develop deformities of the hips in subsequent years that make walking difficult or impossible. This is borne out by the observation of a large group of children with myelomeningocele who over the years have demonstrated muscle imbalances about the hip joints leading to lumbar lordosis, hip deformities, subluxations and dislocations of significant magnitude (Kilfoyle et al., I965, Sharrard, 1964). On the other hand unnecessary, poorly planned and repeated surgery of the hips may lead to complications more severe than if nothing were done.

The purpose of this communication is to give the findings in this group of patients, describe the effects of myelomeningocele on the hip joints, discuss the surgical treatment to restore muscle balance and analyse the results of surgical treatment.

The neurological function in the child with myelomeningocele varies considerably. While it is true that many of these children have distinct levels of neurological deficit, incomplete lesions are very common. Sharrard (I969) has described in detail the course of events that takes place in the hip joint associated with various neurological levels of the lumbar spine. The high incidence of complete or incomplete paralysis of the gluteal muscles causes a muscle imbalance around the hip characterised by greater strength of the hip flexors and adductors. Deformity of the hip in the growing child occurs because of imbalance. The type and severity of these deformities are dependent upon the degree of imbalance.

Iliopsoas muscle transfer was described by Mustard (1959) and Sharrard (1964) and the treatment of paralytic conditions about the hip in poliomyelitis. The indications, technique, and management have been modified by Sharrard (1964), Menalaus (I969), Hayes, et al. (1964) and Cruess and Turner (1970), for the patient with myelomeningocele. The purposes of these operations were to remove a deforming force and to provide better muscle balance around the hip 
joint leading to a stable and mobile hip joint with increased abductor power and improved gait.

\section{MATERIAL}

Sixty-one iliopsoas transfers in 33 myelomeningocele patients were performed. The total population of patients with myelomeningocele from our clinic number I50. The results of hip transfers in these patients have been studied in detail. All patients were born with myelomeningocele and all had neurological deficits characterised by muscle imbalance around the hip with hip flexors and adductors overpowering all other muscles.

The age range at the time of surgery was $I$ to 9 years and 2 months. There were I 5 males and I 8 females. Twenty-eight of the patients had bilateral transfers. The average time between transfers was I7 days. Except for two patients the range was 5 days to 35 days between transfers. These two patients had asymmetric motor function in the lower extremities and iliopsoas transfers were not felt to be indicated on the opposite hips until 22 and 28 months later when transfers were carried out on the opposite hips. All patients were available for continued observation except one who died 6 months following surgery of unrelated causes.

Twenty-six of these patients had hydrocephalus treated with shunt procedures. Twenty of the patients had undergone urinary diversion with uretero-ileal conduit. All of the patients except six had closure of their myelomeningocele defects during the first week of life and most on the first day of life.

Close evaluation of each patient was made repeatedly to establish the neurological function. This neurological evaluation can be done at any age and has proved of great value in predicting progression of deformity, planning for future treatment, and determining functional goals.

An analysis of the status of walking and bracing was made before surgical treatment and on subsequent examinations. Comparisons were made of hip deformity, range of motion, hip stability, muscle function, femoral neck deformity, and complications.

\section{INDICATIONS FOR ILIOPSOAS TRANSFER}

Sharrard (1964) has classified his patients into six groups according to nerve innervation to the lower limbs. This innervation varies considerably but the most important determinant to the development of the hip joint is the status of the balance of muscle strengths.

Group I. Patients in this group had complete paralysis of the lower limbs. Coxa valga was common finding and tightness of the iliotibial band was frequently seen. No patients in this group had iliopsoas transfer.

Group II. These patients had active hip flexion and adduction. Strengths of muscle function were not normal but the absence of other muscle activity created an imbalance. Hip flexion and adduction deformity with coxa valga was common and increased with age. Sixteen of these hips had iliopsoas transfers.

Group III. Thirty-one limbs showed good or normal hip flexion and adduction. All of the hips dislocated before I year of age and many were dislocated at birth. These children whose hips were dislocated at birth usually had severe 
hip flexion deformities with extension deformities of the knees. Valgus and anteversion of the neck of the femur was common. The acetabulum was usually normal radiographically, during the first year or two of life. All these hips had iliopsoas transfers.

Group IV. In this group all of the lumbar nerve roots were functioning. Abduction of the hips and sometimes flexion of the knees functioned actively but weakly. Flexion deformity, subluxation, poor acetabular coverage occurred in all limbs. Twelve had iliopsoas transfers. Dislocation occurred at age 9 in one hip.

Group V. One patient showed only weakness of extension about the hips. Hip flexion deformity was present and both hips were subluxed. Iliopsoas transfers were done bilaterally at about 3 years of age.

Group VI. Patients showing one normal limb were included in this group by Sharrard. No hip abnormalities were observed in this study.

It was evident that when muscle imbalance was greatest or when hip flexors and adductors showed the greatest strength differential over the gluteal muscles dislocation was more frequent and occurred earlier. With milder imbalance dislocation occurred later or subluxation or hip flexion deformity occurred instead (Table I).

\section{TABLE I}

Position of femoral head before surgery

\begin{tabular}{|l|c|c|}
\hline & Dislocated & Subluxed \\
\hline Group II & 8 & 8 \\
Group III & 3 I & - \\
Group IV & I & I I \\
Group V & - & 2 \\
\hline & 40 & 2 I \\
\hline
\end{tabular}

Diagnosis of dislocation of the hip in the patients was made radiographically and clinically. Positioning the hip in extension, abduction, and internal rotation usually reduced the hip, which, if flexed and adducted was completely dislocated. It was usually simple to reduce and dislocate the hip with paralytic dislocation. As long as the limb was held in abduction, extension and internal rotation hip reduction and stability existed.

Patients who demonstrated this imbalance resulting in hip deformity, subluxation, dislocation, or lumbar lordosis underwent iliopsoas transfer. When iliopsoas transfer was indicated the optimum age was 12 to 18 months of age. The children in this age-group were large enough and they could be expected to begin standing and progress to walking at this time of life. In addition chances 
for better development of the hip joint were greater. Transfers were indicated in older children, but operation at an earlier age was desired.

\section{TECHNIQUE OF OPERATION}

The technique of surgery is described elsewhere (Freehafer et al., 1972).

Modifications of this procedure have to be undertaken at certain times. A subcutaneous adductor tentotomy was done as indicated prior to the iliopsoas transfer when adduction contracture existed. When hip flexion deformity was severe the rectus femoris insertion was incised and the rectus femoris and the sartorius were placed in relaxed positions with deformity corrected and not reinserted anatomically. Occasionally it was noted that the atrophic tensor fascia femoris caused hip flexion deformity requiring transverse incision.

The capsule of the joint was ordinarily not opened. Only in situations where a shelf arthroplasty was performed was this carried out. The method of the bone shelf operation was that described by Heyman (1963). It was done only in children aged 3 or over and when the coverage of the femoral head was not sufficient.

Varus osteotomy was performed rarely. The correction of coxa valga is not necessary in the younger children but after the age of 3 or 4 , varus osteotomy may be indicated for severe coxa valga. The upper femur was osteotomised in the subtrochanteric region and two Steinman pins were inserted from the trochanter into the femoral neck and incorporated in the spica cast holding the fragments in the desired position.

Following surgery the patient was immobilised in a bilateral hip spica with the lower extremities in internal rotation, abduction and extension. Both lower extremities were completely incorporated in plaster because the paralysed child often had insufficient control of his extremities. This avoided injury and permitted the child to stand. Length of immobilisation in plaster varied considerably. The younger children who had only iliopsoas transfer were usually allowed out of plaster by 3 or 4 weeks. Over the age of 3 years children were immobilised for a period of 6 weeks following the transfer. However, when a bone shelf was constructed or varus osteotomy was performed, 8 weeks was usually necessary. When hip instability existed immobilisation was extended. Two or 3 days following surgery, the patients were allowed to stand in the hip spicas. A practical routine used was to allow standing for meals and play and to interrupt this with periods of rest in the morning, afternoon and at night time. Figures I, A and B and 2, A-C demonstrate the techniques and result.

\section{RESULTS}

The goals of hip surgery were to correct deformity, provide stable reduced hip joints with normal range of motion, improve hip abduction and extension so that walking ability could be improved.

In order to evaluate results, a number of factors had to be considered (Table II). The most important was the ability to walk. None of the children had normal gait and never will because of their neurological deficit. Independence of walking with or without crutches with braces was the hoped for goal.

Observations of large numbers of myelomeningocele children clearly shows that those with hip dislocations and deformities usually walk poorly and often 
become confined to wheel chairs at an early age. The patients in this series did demonstrate improvement in their walking following iliopsoas transfers and correction of deformities. It is not fair, however, to say that all children improved because of surgery, because many were too young to walk when transfer was performed. However, as children were observed for long periods, it was obvious that gait was better, and bracing less extensive than in unoperated children who had deformities and dislocations of their hips.

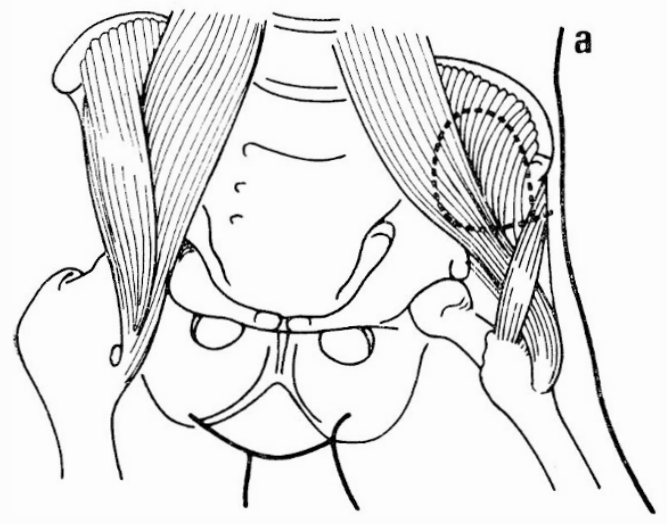

FIG. I

A, Diagram showing transferred iliopsoas muscle and opening made in iliac crest. $\mathrm{B}$, Diagram showing skin incision used for iliopsoas transfer.
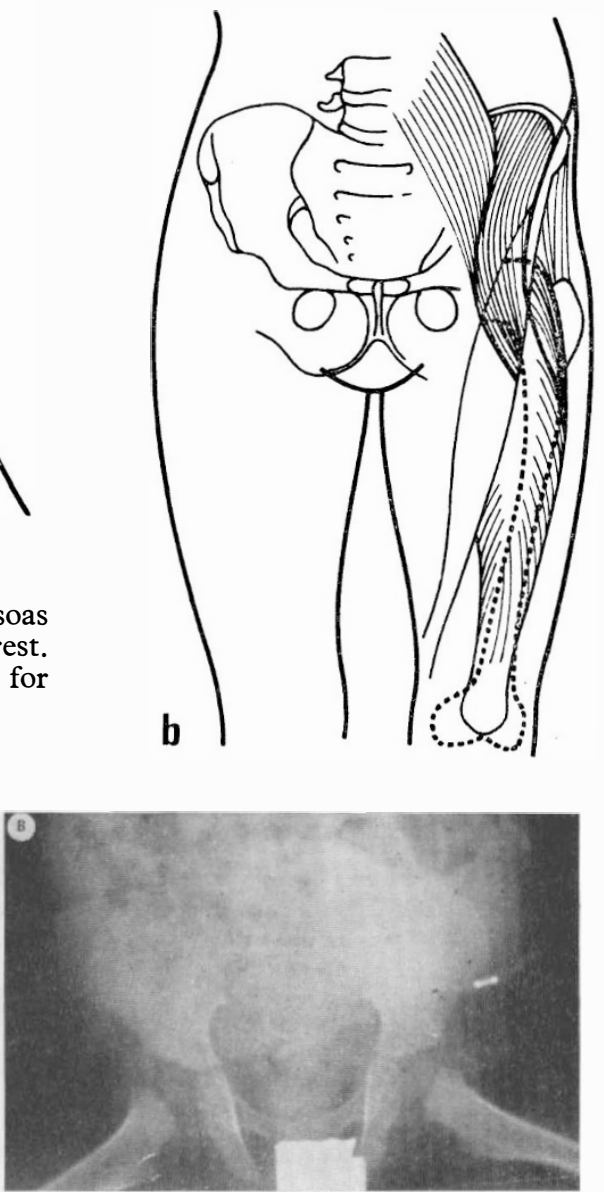

FIG. 2

A, Pre-operative hip roentgenograms of patient in Group III. Both hips dislocated on flexion and adduction. B, Frog leg laterals of both hips of same patient. C, Hips of same patient three years after iliopsoas transfer. 
Table III lists factors which determine walking ability. A child with active hip flexion and all muscles innervated at segments above can learn to walk well with braces and crutches. Any one or a combination of factors listed may make walking impossible. In evaluating the patients in this series all of these factors were considered.

Some are not apparent at an age when iliopsoas transfer is indicated. Intelligence is difficult at best to determine. Parental guidance is often impossible to evaluate and parents of these children often change. Evaluation of growth and development can be done only by observation over a period of years. While it is desirable to understand these factors in children undergoing surgery it is not

\title{
TABLE II
}

Factors used in evaluation of iliopsoas transfers

\author{
Walking ability \\ Bracing \\ Muscle strength \\ Stability \\ Range of motion \\ Coxa Valga \\ Acetabular index \\ Anteversion \\ Complications
}

\section{TABLE III}

Factors which determine walking ability

Remaining muscle strength

Associated deformities

Intelligence

General growth and development

Parental involvement

Psychological factors

always possible if surgery is to be performed shortly after I year of age at a time which is optimal.

All patients in this series improved their gait as they grew older except one whose hip surgery was satisfactory but who was handicapped by poor general development, weakness, mental retardation, scoliosis, and behavioural problems. Some of the patients had not begun to walk when iliopsoas transfer was performed but it was observed that walking in children who had iliopsoas transfers was better than gait of comparable children who did not have surgery.

Children with associated deformities and those who had the greater number of operative procedures were delayed somewhat in their development. The others have either undergone corrective surgery (Table IV), had corrective plaster casts, or been controlled with bracing. The type and incidence of deformities are listed in Table $\mathrm{V}$.

As a routine children who reach I year of age received braces and began standing. If iliopsoas transfer was done they received braces and began standing after surgery when the plaster casts were removed. Most children began with long 
leg braces with double uprights, pelvic band with hip locks, droplocks at the knees (or no joints if too small) and ankle stops to allow $20^{\circ}$ dorsiflexion and $10^{\circ}$ plantar flexion. As they grew and developed they learned to stand independently in the crib or by a chair or holding on to parents. At about 2 years of age most intelligent children can learn to use Canadian crutches. The four-point gait, swing to, or swing through can be learned by these children and their parents but the fourpoint gait was emphasised. The greater the delay in providing braces and crutches

\section{TABLE IV}

Other orthopaedic procedures performed

$\begin{array}{lr}\text { Yount fasciectomy } & 4 \\ \text { Tibial osteotomy } & \text { I } \\ \text { Spinal fusion } & \text { I } \\ \text { Grice bone block } & 4 \\ \text { Foot capsulotomy and heel cord lengthening } & 35 \\ \text { Tibialis anterior transfer to heel cord } & 8 \\ \text { Tibialis posterior transfer to dorsum of foot } & \text { I } \\ & -54\end{array}$

TABLE $\mathrm{V}$

Associated deformities

Spine

$\begin{array}{lr}\text { Scoliosis } & 3 \\ \text { Lumbar kyphosis } & \text { I } \\ \text { Thoracolumbar kyphosis } & \text { I } \\ \text { Knee } & \\ \text { Flexion contracture } & \text { I2 } \\ \text { Recurvatum } & 9 \\ \text { Valgus } & 3 \\ & \\ \text { Foot } & 27 \\ \text { Equinovarus } & 7 \\ \text { Calcaneus or calcaneovalgus } & 2\end{array}$

the longer it took for these children to walk independently. The rapidity and success in walking was very closely related to the factors in Table III.

As the children in this series improved the hip locks became unnecessary. Patients in Groups III, IV and V often improved rapidly and components of their bracing could be reduced by elimination of hip locks and then pelvic band and knee locks until only short leg braces were necessary. Reducing the amount of bracing should be done cautiously. If the children do well all is fine, but, occasionally in the process of brace reduction the patient's gait will suffer or loss of support will lead to added stress to the knees or loss of rotary control of the lower limbs will be undesirable.

Muscle strength of all innervated functioning muscles was very important in determining success in walking. They were all able to stand and walk but not as well as desired. The strength of the iliopsoas muscle was also important. It 
was impossible to grade strengths before and after transfer because of age. A general impression was obtained and the presence of activity was determined. The measure of success in iliopsoas transfer was not related to its strength anyway. The removal of a deforming force probably was as important as the new muscle activity after transfer although good strength was desirable.

All muscle transfers functioned except one. One showed no voluntary activity before or after surgery. A transfer was done because of the dislocation and because electrical stimulation showed activity in his hip adductors and flexors. The hip is still dislocated and it is doubtful that iliopsoas transfer was indicated.

At surgery when the iliopsoas muscle was transferred stability of the femoral head within the acetabulum was improved. However, some were less stable than others especially if adducted and flexed. Six hips were unstable after transfer unless the femurs were held in abduction, extension, and internal rotation. Two of these patients' hips dislocated after removal of plaster. Two patients were immobilised in plaster for a total of 4 months. One child crawled out of three wellfitting hip spicas and the last attempt was made with Steinman pins through the femurs incorporated in plaster. Ten hips underwent intracapsular bone shelves because of poorly formed acetabulae. This combined with iliopsoas transfers led to stable hips.

Good motion of the joints of the lower limbs of myelomeningocele patients. is extremely important. The impairment of sensation along with limited joint motion favours the development of neurotrophic joints. This complication has not been seen in this group of patients but would not be anticipated so early. However, several adults under observation who had triple arthrodesis, knee fusion, or stiff hips as children have developed neurotrophic changes.

The acetabular indices in the infants were generally normal. Patients with hip muscle imbalance showed failure of normal acetabular development as they passed 3 years of age. This was manifested by incomplete femoral head coverage.

The neck shaft angles improved following iliopsoas transfer. The majority of the hips had a coxa valga which was more severe if the hip was dislocated. The average correction while under observation was $20^{\circ}$ for the hips that were dislocated before transfer and $10^{\circ}$ for the hips that were subluxed.

Roentgenograms taken to estimate the amount of anteversion showed that there were only three hips with retroversion at follow-up. Two of these hips had varus derotational osteotomies and the third retroverted femoral head occurred when the patient sustained bilateral intertrochanteric fractures after cast removal for a left iliopsoas transfer.

The severity of the anteversion paralleled the coxa valga and therefore the dislocated hips usually had the most severe anteversion. The degree of anteversion was often fully appreciated at the time of transfer when maintenance of reduction could be achieved only with considerable internal rotation.

Only two of the patients had more than one major operative procedure on a hip. Great effort was made to plan treatment so that multiple surgery could be avoided. The authors believed that repeated surgery would certainly lead to stiffness and a poor result.

Table VI gives the location and numbers of fractures that occurred. Most took place early in the series and were observed in the first few days after plaster removal. Elevation of temperature, local heat, swelling locally and throughout the extremity were common findings and suggested the diagnosis which was confirmed by roent- 
genograms. Treatment usually consisted of careful positioning of the extremities and when swelling receded braces were used. In I to 2 weeks most fractures were healed sufficiently to permit standing in braces and progression to gait training. All fractures healed promptly with no complications except one who has a deformity of the femoral head and neck.

With experience it became routine to stand all children regularly while still in hip spicas. On removal from plaster extreme caution was exercised to avoid twisting or forceful motions. Two persons carried or lifted the children and changed diapers. No passive range of motion was allowed unless it was in water with gravity. Early application of braces to protect the extremities was helpful in reducing external forces (Drennan and Freehalfer, I97I).

\section{TABLE VI}

Fractures after cast removal (6 patients)

$\begin{array}{lr}\text { Femur } & \\ \text { Neck } & 2 \\ \text { Intertrochanteric } & 2 \\ \text { Subtrochanteric } & 2 \\ \text { Shaft } & 0 \\ \text { Supracondylar } & 4 \\ \text { Tibia } & \\ \quad \text { Proximal } & 2 \\ \text { Shaft } & 0 \\ \text { Distal } & 2 \\ & - \\ & \text { I4 }\end{array}$

TABLE VII

Causes for poor hip results

Persistent dislocation

Limited motion

$\begin{array}{ll}\text { Scoliosis and poor development } & 2 \\ \text { Deformity of head and neck } & \text { I }\end{array}$

One hip developed a wound infection. This occurred 3 months after operation when the child was at home and at a stage when he was standing in braces. His symptoms were similar to those seen with the fractures but none were found. Incision and drainage was performed, antibiotics administered and the infection resolved with no further problems.

The goals of iliopsoas transfer were to correct deformity, to provide a stable reduction, to maintain a good range of motion and to improve the strength of abduction and extension. This was accomplished in all but six patients (nine hips). The failures are shown in Table VII.

\section{DISCUSSION}

The management of the patient with myelomeningocele is difficult. The many and varied medical problems require a variety of specialists all of whom can 
be overwhelmed by their complexity. The social, psychological, physical, educational and vocational guidance of these children and their families demands expert assistance. As the adult population of these patients increases other specialists will have to play an enlarging role.

The hip and its abnormalities represent only a small part in this total picture. To perform an operation which is unsuccessful may be catastrophic. To neglect a deformity on the other hand may be equally harmful.

The author believes that muscle imbalance around the hip joint leads to deformity whether dislocation, subluxation, flexion-adduction contracture, or lumbar lordosis is disabling and will become increasingly so with age. It is realised that multiple or unsuccessful hip surgery may be disabling as well, but the majority of hips in this series were converted to stable and mobile hips with increased active function resulting in improved gait. While anticipated goals for some children were not reached, surgery on the hips was not usually the primary reason.

Myelomeningocele patients with paralytic hip deformities manifest unique problems. Much has been written about congenital dislocation of the hip, poliomyelitis with paralytic deformities of the hip, cerebral palsy, arthrogryposis and others but all are different. One cannot apply the principles of treatment of these conditions to that of myelomeningocele.

The loss of sensations of position, vibration, touch, pain is only one reason. Bone in myelomeningocele patients is soft compared to bone seen in other conditions. Dislocation of a hip and its reduction in myelomeningocele is almost always simply done by positioning of the extremity. One can easily be fooled if he sees a normal roentgenogram with the hips in abduction, extension, and internal rotation and neglects to examine the hips in adduction and flexion.

In patients with myelomeningocele skin traction in an asensory limb usually leads to catastrophic skin breakdown. Skeletal pin traction is attended by rapid bone resorption necessitating early pin removal. Use of the frog leg position causes severe persistent contracture. The use of plaster immobilisation fails to correct muscle imbalance and hips promptly redislocate. All of the children over 3 with dislocated hips came to our clinic having previously received the usual treatment for congenital dislocation of the hip and they were all dislocated. Fortunately, most of them were improved following correction of muscle imbalance but the authors believe that their results would have been much better had iliopsoas transfer been carried out at an earlier age.

Most of our children in the clinic have had multiple fractures at one time or another from a variety of minor trauma. When immobilised in plaster casts, bones atrophied further and in the presence of abnormal muscle and sensory control the incidence of fractures was markedly increased. It is very important to realise that traction, continued cast immobilisation and other conventional methods may be harmful to the myelomeningocele patient (Drennan and Freehafer, I97I).

The author believes that the treatment that has been provided for muscle inbalance of the hip in patients with myelomeningocele has led to marked improvement in most patients. It is not easy and requires an organised team approach. Many orthopaedists will not want to attempt this kind of treatment and this is justified if facilities and assistance of many specialists are not available. 


\section{SUMMARY}

Patients with myelomeningocele pose many problems. Muscle imbalance about the hip described in this communication is only one. The correction of this imbalance by removing a deforming force and using the iliopsoas transfer to provide active abductor power and greater stability of the hip usually resulted in improved function and better gait.

The problems of myelomeningocele are sometimes overwhelming and require proper facilities and specially trained personnel. It is a unique medical disorder and deformities of the hip must be treated as such. The application of principles learned for the treatments of other hip deformities do not apply.

\section{REFERENCES}

Cruess, R. L. \& TuRner, N. S. (1970). Paralysis of the hip abductor muscles in spina bifida. Results of treatment by mustard procedure. F.B.F.S. 52A, I364-1372.

DrenNan, J. C. \& FreEhafer, A. A. (I97I). Fractures of the lower extremities in paraplegic children. Clin. Orthop. 77, 2 I I-2 I7.

Freehafer, A. A., Vessely, J. C. \& MACK, R. P. (1972). Iliopsoas transfer in the treatment of myelomeningocele patients with paralytic hip deformities. F.B.F.S. 54 A, I715-I729.

HAyes, J. T., Gross, H. P. \& Dow, S. (1964). Surgery for paralytic defects secondary to myelomeningocele and myelodysplasia. F.B.F.S. 46A, I577-1597.

Heyman, C. H. (1963). Long-term results following a bone shelf operation for congenital and some other dislocations of the hip in children. F.B.F.S. 45A, I I3-I I 46.

Kilfoyle, R. M., Foley, J. J. \& Norton, P. L. (1965). Spine and pelvic deformity in childhood and adolescent paraplegia. F.B.F.S. 47A, 659 .

Menelaus, M. D. (1969). Dislocation and deformity of the hip in children with spina bifida cystica. F.B.F.S. $5 \mathrm{IB}, 238-25 \mathrm{I}$.

MUSTARD, W. T. (I952). Iliopsoas transfer for weakness of the hip abductors. F.B.F.S. 34A, 647-650.

MUSTARD, W. T. (1959). A follow-up study of iliopsoas transfer for hip instability. F.B.F.S. $4 \mathrm{IB}, 289-298$.

SHARRARD, W. J. W. (I964). Posterior iliopsoas transplantation in the treatment of paralytic dislocation of the hip. F.B.F.S. 46B, 426-444. 